\title{
Strong Linear Correlation Between Eigenvalues and Diagonal Matrix Elements
}

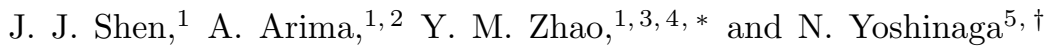 \\ ${ }^{1}$ Department of Physics, Shanghai Jiao Tong University, Shanghai 200240, China \\ ${ }^{2}$ Science Museum, Japan Science Foundation, 2-1 Kitanomaru-koen, Chiyoda-ku, Tokyo 102-0091, Japan \\ ${ }^{3}$ Center of Theoretical Nuclear Physics, National Laboratory of Heavy Ion Accelerator, Lanzhou 730000, China \\ ${ }^{4}$ CCAST, World Laboratory, P.O. Box 8730, Beijing 100080, China \\ ${ }^{5}$ Department of Physics, Saitama University, Saitama 338-8570, Japan
}

(Dated: October 24, 2018)

\begin{abstract}
We investigate eigenvalues of many-body systems interacting by two-body forces as well as those of random matrices. We find a strong linear correlation between eigenvalues and diagonal matrix elements if both of them are sorted from the smaller values to larger ones. By using this linear correlation we are able to predict reasonably all eigenvalues of given shell model Hamiltonian without complicated iterations.
\end{abstract}

PACS numbers: 21.10.Re, 21.10.Ev, 21.60. Cs

Keywords:

Diagonalization of matrices is a very common practice in many fields. There have been a number of algorithms for diagonalization, such as the Jacobi method, the Householder method, the Lanczos method, etc. [1]. Full diagonalizations of obtaining all the eigenvalues become difficult when the dimension of matrices is larger than $10^{5}$. One can obtain a very few lowest eigenvalues of sparse matrices when dimension of matrices is $10^{8-10}$ by super computers. There have been also many efforts in obtaining eigenvalues by using the energy centroid and spectral moments, and typical works along this line can be found in, e.g., Refs. [2, 3, 4, 5, 6]. Unfortunately, the eigenvalues such obtained by using centroids and moments are valid at the statistical level (i.e., after the ensemble average) and not good enough for individual diagonalizations.

The purpose of this Letter is to report a remarkable correlation between eigenvalues and diagonal matrix elements, if both are sorted from smaller values to larger ones. One can predict approximately all eigenvalues of given matrices by using this correlation. Although eigenvalues such predicted are not exact, they are good approximations of exact results (within a precision of $1-4 \%$ in examples that we have studied).

Let us begin with Hamiltonian matrices which conserve the angular momentum. The two-body interaction parameters are taken to be Gaussian random numbers (i.e., two-body random ensemble [7] which is called the TBRE). In such cases both the diagonal matrix elements of a many-body system and their eigenvalues are known to exhibit Gaussian distributions approximately. One easily conjectures that there might exist a linear correlation between these two sets of quantities. Let us denote exact eigenvalues obtained by diagonalization by using $E_{i}^{\text {exact }}$, dimension of matrix by $D$ (number of

*Electronic address: ymzhao@sjtu.edu.cn

${ }^{\dagger}$ Electronic address: yoshinaga@phy.saitama-u.ac.jp spin $I$ states is denoted by $D_{I}$ ), predicted eigenvalues by $E_{i}^{\text {exact}}$, diagonal matrix elements by $H_{i i}$, and average energy by $\bar{H}$. We define the linear correlation coefficient $r=\frac{\sum_{i}\left(H_{i i}-\bar{H}\right)\left(E_{i}^{\text {exact }}-\bar{H}\right)}{\sqrt{\sum_{i}\left(E_{i}^{\text {exact }}-\bar{H}\right)^{2} \sum_{i}\left(H_{i i}-\bar{H}\right)^{2}}}$. The absolute value of $r,|r|$, is less than or equals to 1 . If there exists a strong linear correlation between $E_{i}^{\text {exact }}$ and $H_{i i}$ $(i=1,2, \cdots n),|r| \rightarrow 1$. We investigate below whether or not the coefficient $r$ is very close to 1 by a number of different configurations. We take six successive sets of random interactions for each system in demonstrating the correlation between eigenvalues and diagonal matrix elements.

In Fig. 1(a-f), we present exact eigenvalues versus diagonal matrix elements of $I=20$ states (dimension $\left.D_{I}=29\right)$ for four identical fermions in a single- $j\left(j=\frac{31}{2}\right)$ shell. Both eigenvalues and diagonal matrix elements are sorted from the smallest to the largest. The linear correlation coefficients $r$ are more than 0.95 in all these panels. Each panel corresponds to one set of random interaction parameters.

In Fig. 2(a-f), similar results are presented for $s d$ boson systems [8]. Here $I=6$ and boson number $n=12$ (dimension $D_{I}=37$ ). In Fig. 3(a-f) we present results for the $I=17\left(D_{I}=508\right)$ states of three valence protons and three valence neutrons in the $\left(2 s_{1 / 2}, 1 d_{3 / 2}, 0 i_{11 / 2}\right)$ shell; in Fig. 4 we present results of $I=4\left(D_{I}=3017\right)$ states of the same configuration. The Hamiltonian that we use in Figs. 3-4 conserves parity and isospin as well as angular momentum, i.e., here we use the conventional shell model Hamiltonian in nuclear structure physics. The two-body interactions are taken to be random values following the Gaussian distribution. Correlations in all these figures are seen to be striking, although there are small deviations when eigenvalues are around the smallest or the largest.

Now we discuss how one obtains the predicted linear correlation by a simple algorithm. Let us assume that

$$
E_{i}=A H_{i i}+B
$$


where $E_{i i}$ is the eigenvalues of the Hamiltonian. By using

$$
\begin{aligned}
& \sum_{i=1}^{D} E_{i}=\sum_{i=1}^{D} H_{i i}, \\
& \sum_{i=1}^{D}\left(E_{i}\right)^{2}=\sum_{i, j=1}^{D} H_{i j}^{2},
\end{aligned}
$$

we obtain

$$
\begin{aligned}
A=\sqrt{\frac{D \sum_{i=1}^{D} \sum_{j=1}^{D} H_{i j}^{2}-\left(\sum_{i=1}^{D} H_{i i}\right)^{2}}{D \sum_{i=1}^{D} H_{i i}^{2}-\left(\sum_{i=1}^{D} H_{i i}\right)^{2}}} \\
=\sqrt{\frac{\overline{H^{2}}-\bar{H}^{2}}{\sum_{i} H_{i i}^{2} / D-\bar{H}^{2}}}, \\
B=(1-A) \bar{H} .
\end{aligned}
$$

All straight lines in this paper are plotted by using the above relation.

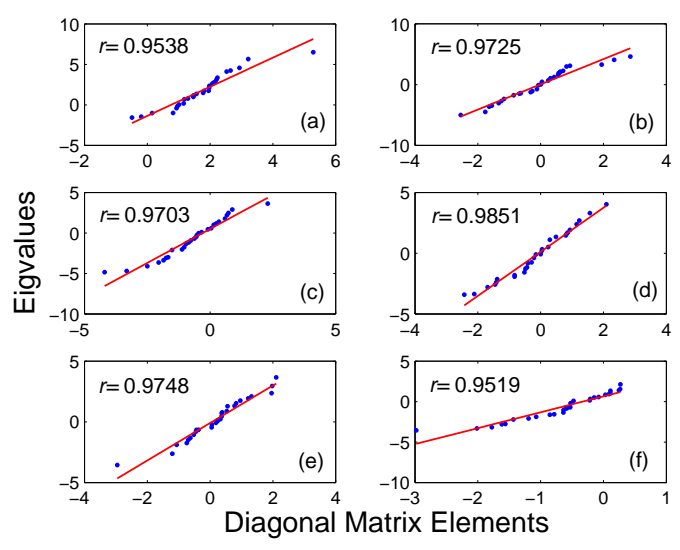

FIG. 1. (Color online) Linear correlation between exact eigenvalues and diagonal matrix elements. These examples correspond to four fermions in a single- $j$ shell with $j=31 / 2$. Here $I=20, D_{I}=29 . \quad r$ is the linear correlation coefficient. Straight lines are plotted by using Eq. (1), with $A$ and $B$ values evaluated by Eq. (3).

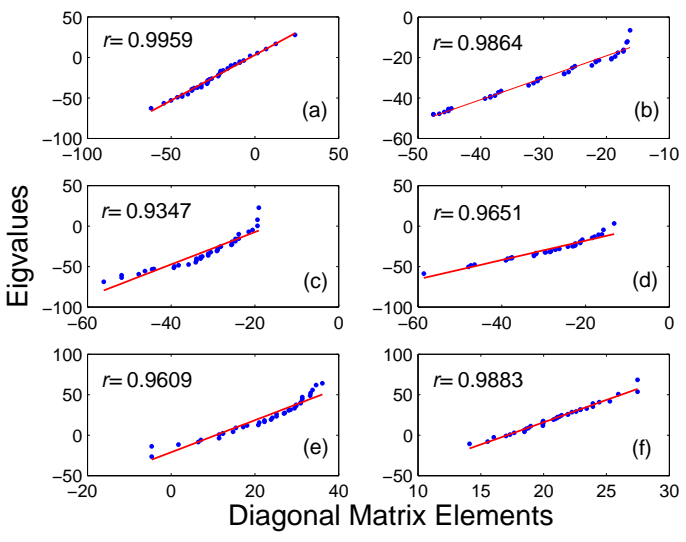

FIG. 2. (Color online) The same as Fig. 1 except that these examples correspond to $s d$-boson systems. Here boson number $n=12, I=6, D_{I}=37$.
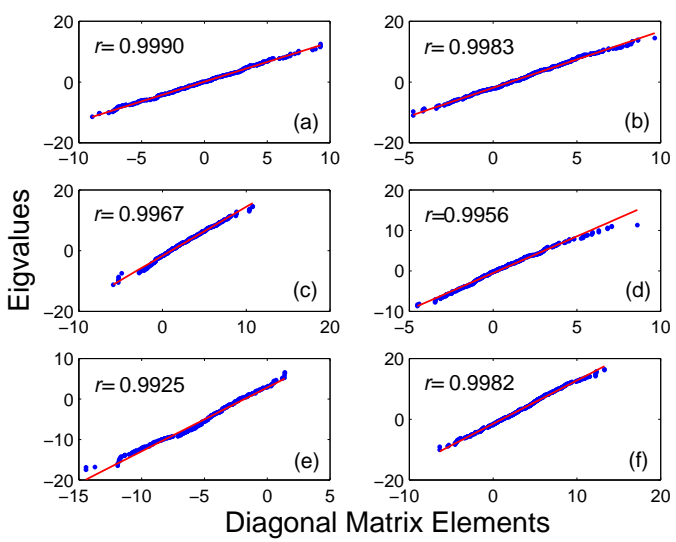

FIG. 3. (Color online) The same as Fig. 1 except that these examples correspond to three valence protons and three valence neutrons in the $\left(2 s_{1 / 2}, 1 d_{3 / 2}, 0 i_{11 / 2}\right)$ shell. Here $I=17$ and $D_{I}=508$.

In the above examples the two-body interacting parameters are given by Gaussian random numbers. The matrices of these systems are given by coefficients of fractional parentage determined by geometry of the configuration and two-body interactions which are taken to be Gaussian. Now we come to matrices in which all matrix elements are given by random numbers.

In the first set of examples, all matrix elements are taken to be uniformly distributed random numbers between -1 and 1 . Figure 4 shows exact eigenvalues versus diagonal matrix elements (dimension $D=500$ ), both sorted from smaller to larger values. The correlation is seen to be very good, except for small deviations near the largest and the smallest eigenvalues.
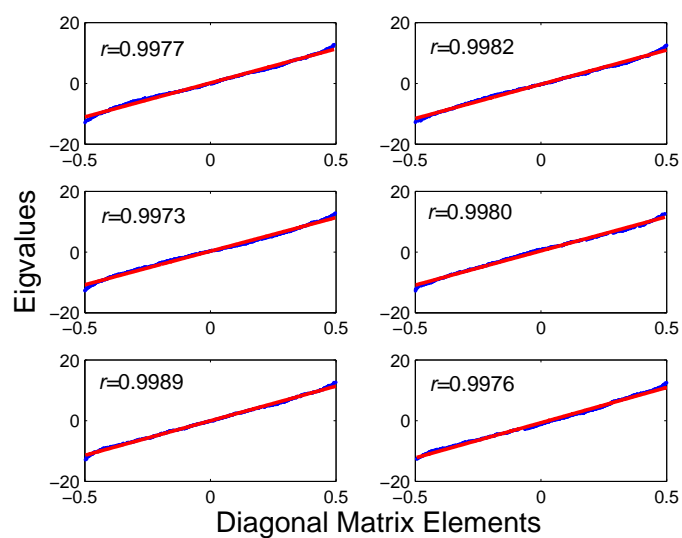

FIG. 4 (color online) Eigenvalues versus diagonal matrix elements $\left(d_{I}=500\right)$. Both eigenvalues and diagonal matrix elements are sorted from smaller values 
to larger ones. All matrix elements are taken to be uniformly distributed random numbers. One sees that the eigenvalues predicted by linear correlation and the exact values are almost equal except for a very few around the maximum and minimum values.

We also investigate the emergence of the above correlation in matrices for which all matrix elements are random numbers following the Gaussian distribution. Figure 5 shows results of matrices with $D=500$ and all matrix elements being Gaussian random numbers. The correlation seems not linear here, but rather a hyperbolic tangent function because eigenvalues in these case follow Wigner's semicircle distribution. However, it is important to point out two important facts. (I) There also exists a remarkable linear correlation between eigenvalues and diagonal matrix elements on average in these cases. The above linear correlation is actually very good except for eigenvalues near the smallest or largest ones, because the level density of eigenvalues in the middle part is much larger than that near the minimum or maximum. All "medium" eigenvalues can be predicted very well by the linear correlation determined by Eqs. (1-3). For matrices with dimension larger than 20, correlation coefficient $r$ is larger than 0.95 . We note without details that one easily obtains the hyperbolic tangent function which seems proper in fitting the results around the minimum or maximum eigenvalues, by using matrix elements in the same way as we do for the TBRE systems. (II) The correlation coefficients between eigenvalues and diagonal matrix elements are larger than 0.995 if one artificially enlarges diagonal matrix elements by about two orders or more (typically by a factor of 50-60) for Gaussian random matrices. The latter fact is very important, because in the nuclear shell model calculations, magnitudes of diagonal matrix elements are usually much larger than (non-zero) off-diagonal matrix elements by about one or two orders.

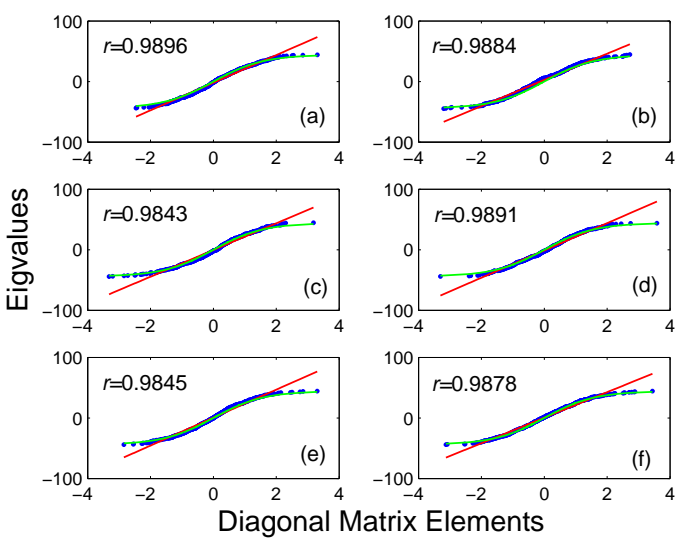

FIG. 5. (Color online) Correlation between eigenvalues of random matrices (Gaussian type) and diagonal matrix elements. The dimension is 500 here. It is noted that the linear correlation is actually very good except for eigenvalues near the smallest or largest ones, because the level density of eigenvalues in the middle region is much larger than that of eigenvalues near the minimum or maximum. All $r$ values are larger than 0.98.

Now we exemplify our approach by a realistic example. In Fig. 6(a), we present eigenvalues of spin $I=0$ states of ${ }^{24} \mathrm{Mg}\left(D_{I}=1161\right)$ versus the diagonal matrix elements of the same configurations. Here we use the USD interactions [9]. It is seen that the linear correlation is very good. Similar results can be found for other spin states. In Fig. 6(b) we show exact eigenvalues $E_{i}^{\text {exact }}$ in comparison with those predicted by using linear correlations $\left(E_{i}\right.$ in Eq.(1)). Although eigenvalues predicted by using the linear correlation deviates from the exact values slightly, they are close to the exact ones in general. More important, the agreement between predicted eigenvalues and exact results is substantially improved when one goes to the medium region. As shown in Fig. 6 (c), predicted 100 th- 105 th $0^{+}, 2^{+}, 3^{+}, 4^{+}, 6^{+}$states are very close to exact eigenvalues obtained by diagonalizations.
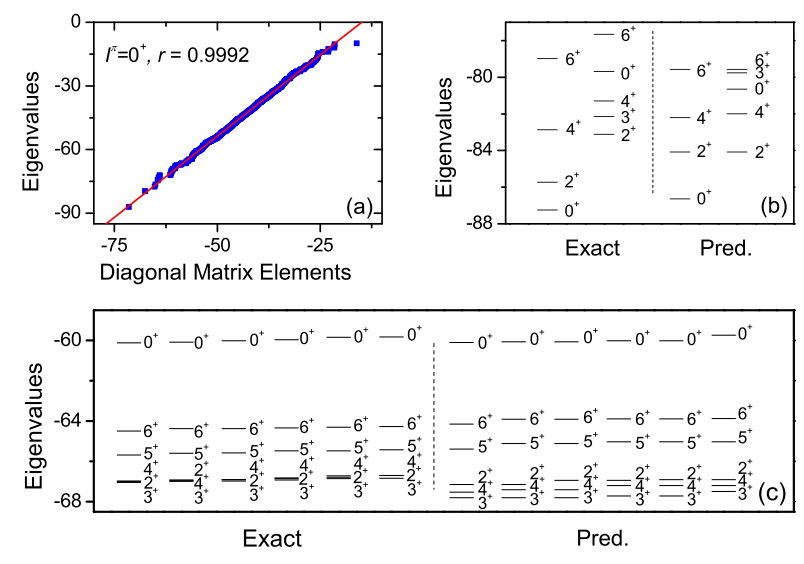

FIG. 6 Energy Spectrum for ${ }^{24} \mathrm{Mg}$. The USD interactions are used here. (a) Eigenvalues of $I=0$ states obtained by diagonalization versus diagonal matrix elements. One sees a striking linear correlation. The correlation coefficient $r$ is larger than 0.999. (b) Energy spectrum by exact diagonalization and that predicted by linear correlation. The eigenvalues of the lowest two $I=2$ levels (and two lowest $I=6$ levels) are degenerate because diagonal matrix elements corresponding to these states are equal due to the isospin conservation of the USD interactions. (c) Energies of excited states in medium region. The left hand side corresponds to exact energies obtained by diagonalizations, and the right hand side corresponds to predicted values by using linear correlations. The 100th-105th $0^{+}, 2^{+}, 3^{+}, 4^{+}, 6^{+}$states are presented.

To summarize, in this paper we have discovered the 
linear correlation between exact eigenvalues and diagonal matrix elements for individual runs of the two-body random ensemble or random matrices, if both exact eigenvalues and diagonal matrix elements are sorted from the smaller values to larger ones. We exemplify this correlation by fermions in a single- $j$ shell, many- $j$ shells and $s d$ bosons by using random interactions, and energy spectra of ${ }^{24} \mathrm{Mg}$ by using the USD interactions.

This linear correlation seems to be universal. For twobody random ensembles, the linear correlation coefficient is $~ 0.95-1.00$; for matrices in which all matrix elements are uniformly distributed random numbers, the linear correlation is very good; for Gaussian type random matrices, there are sizable deviations between exact eigenvalues and predicted eigenvalues only if eigenvalues are close to the minimum or maximum, and in this case the linear correlation is well applicable to predicting levels in the medium region.

Although eigenvalues obtained here are not exact, they are very good approximations of exact values. We therefore believe our results are very useful in studying problems in which one needs approximate eigenvalues when other methods are not possible.

Acknowledgements: The authors would like to thank Prof. K. Ogawa for modifying his shell model code. Two of the authors (JJS and YMZ) thank the National Natural Science Foundation of China for supporting this work under grants 10575070,10675081 . This work is also supported partly by the Research Foundation for Doctoral Program of Higher Education in China under grant No. 20060248050, Scientific Research Foundation of Ministry of Education in China for Returned Scholars, the NCET-07-0557, and by Chinese Major State Basic Research Developing Program under Grant 2007CB815000.
[1] S. A. Teukolsky and B. P. Flannery, Numerical Recipes in C++, William H. Press (1992).

[2] K. F. Ratcliff, Phys. Rev. C3, 117 (1971).

[3] F. J. Margetan, A. Klar, and J. P. Vary, Phys. Rev. C27, 852 (1983).

[4] V. Velázquez and A. P. Zuker, Phys. Rev. Lett. 88, 027502 (2002).

[5] T. Papenbrock and H. A. Weidenmueller, Phys. Rev. Lett. 93, 132503 (2004); Phys. Rev. C 73, 014311 (2006);

[6] N. Yoshinaga, A. Arima and Y. M. Zhao, Phys. Rev. C 73, 017303(2006); J. J. Shen, Y. M. Zhao, A. Arima and
N. Yoshinaga, to be published.

[7] J. B. French and S. S. M. Wong, Phys. Letters 33B, 449 (1970); 35B, 5 (1971) ; T. A. Brody et al., Review of Modern Physics 53, 385 (1981).

[8] A. Arima and F. Iachello, Ann. Phys. 99, 253 (1976); ibid. 111, 209 (1978); ibid. 123, 468 (1979); for a review, see F. Iachello and A. Arima, the Interacting Boson Model, Cambridge Univ. Press, Cambridge, England, 1987.

[9] B. H. Wildenthal, Prog. Part. Nucl. Phys. 11, 5 (1984). 